Discussion These data provide a basis for further studies of the relationship between sleep and other risk factors. It also provides valuable data about sleep habits in Africa, with potential for future analysis of how it is affected by urbanisation and industrialisation. Finally, it offers a unique opportunity for a population-based comparison of the effects of treated and untreated HIV infection, increasingly unavailable elsewhere.

\section{P059 ADHERENCE TO WAKEFULNESS PROMOTING MEDICATION IN PATIENTS WITH NARCOLEPSY}

Grainne D'ancona*, Laura Perez-Carbonell, Elaine Lyons, Valentina Gnoni, Guy D Leschziner, Brian D Kent. Guy's and St Thomas' NHS Foundation Trust, London, UK

\subsection{6/bmjresp-2019-bssconf.59}

Introduction Excessive daytime sleepiness (EDS) is disabling and it's control is usually contingent on ongoing pharmacological therapy including: modafinil, methylphenidate, dexamfetamine, pitolisant, and sodium oxybate. Irrespective of the medication used, all pharmacological options must be taken to have an effect and while assessment of treatment adherence is standard clinical practice in many chronic conditions, in sleep medicine, evidence regarding adherence to prescribed medications is strikingly limited.

The aim of this study was to assess degree and predictors of adherence to prescribed treatment in patients with narcolepsy attending a tertiary Sleep Disorders Centre.

Methods We examined adherence to treatment in consecutive adult patients with a final diagnosis of narcolepsy by comparing prescription collection rates with prescribed therapy over a one-year period. Three levels of adherence were defined depending on the medication supplied in the last year in

Abstract P059 Table 1 patient characteristics

\begin{tabular}{|l|c|}
\hline & $\mathrm{N}=123$ \\
\hline Age & $39.4( \pm 13.9)$ \\
\hline Female sex & $69(56.1 \%)$ \\
\hline Diagnosis & \\
Narcolepsy type 1 & $99(80.5 \%)$ \\
Narcolepsy type 2 & $24(19.5 \%)$ \\
\hline Psychiatric comorbidities & $28(22.8 \%)$ \\
\hline Refractory sleepiness & $49(39.8 \%)$ \\
\hline Number of wakefulness-promoting & \\
medications at last visit & \\
0 & $7(5.7 \%)$ \\
1 & $67(54.5 \%)$ \\
2 & $34(27.6 \%)$ \\
3 & $13(10.6 \%)$ \\
4 & $2(1.2 \%)$ \\
\hline Patients on & \\
Modafinil & $57(46.3 \%)$ \\
Methylphenidate XL & $34(27.6 \%)$ \\
Methylphenidate IR & $16(13 \%)$ \\
Dexamphetamine & $25(20.3 \%)$ \\
Sodium Oxybate & $38(30.9 \%)$ \\
\hline Good ( $\geq 80 \%)$ & $65(52.8 \%)$ \\
Intermediate (51-79\%) & $15(12.2 \%)$ \\
Poor ( $\leq 50 \%)$ & $43(35 \%)$ \\
\hline
\end{tabular}

proportion to the total prescribed: poor $(\leq 50 \%)$, intermediate $(51-79 \%)$, and good $(\geq 80 \%)$ adherence. Patients with adherence $<80 \%$ were considered as sub-optimally adherent.

Results Demographic and clinical characteristics 162 patients were identified, from which 123 subjects with accurate information regarding current treatment regimen and adherence were included (see table 1). Good adherence was seen in $52.8 \%$ of patients, whilst $12.2 \%$ were intermediately and $35 \%$ poorly adherent. No difference was seen in proportion of good adherence between patients with refractory vs nonrefractory symptoms $(41.5 \%$ vs $58.5 \% ; \mathrm{p}=0.68)$. The proportion of refractory patients with suboptimal adherence was 44.9\%. Patients with NT1 were less likely than those with NT2 to have suboptimal adherence $(40.4 \%$ vs $75 \%$; $\mathrm{p}=0.002)$.

Discussion Our findings suggest that poor adherence is observed in a high proportion of patients with narcolepsy, and that a diagnosis of NT2 seems to be associated with nonadherence to prescribed treatment. Adherence to treatment should be routinely assessed in narcolepsy, particularly prior to initiating any step-up in therapy.

\section{P060 CO-MORBIDITY OF DELAYED SLEEP PHASE AND PSYCHIATRIC CONDITIONS IN A COHORT OF ADOLESCENTS}

Liisa Kuula* , Anu-Katriina Pesonen. University of Helsinki, Helsinki, Finland

\subsection{6/bmjresp-2019-bssconf.60}

Introduction Delayed Sleep Phase (DSP) is an extremely common sleep problem during adolescence. It is typically manifested as frequently occurring late bedtimes and difficulties waking up. The prevalence in entire populations is estimated to be over $15 \%$. While it is common in typically developing teenagers (estimates ranging from 15\%$50 \%$ ), its prevalence may be even higher in those suffering from psychiatric conditions such as severe depression or anxiety.

Methods We studied a cohort sample $(n=295)$ of 17 -year-olds (70\% girls) in order to detect co-morbidity of DSP and several psychiatric disorders. For sleep measurements we used actigraphy (GeneActiv Original) for a minimum of 7 days as well as the Pittsburgh Sleep Quality Index. These served as tools for estimating a tendency towards DSP, which was defined as having a bedtime later than $1 \mathrm{AM}$ for more than three times per week. Psychiatric disorders (major depressive disorder, generalized anxiety disorder, hypo-manic disorders, obsessive-compulsive disorder, psychotic disorders, and eating disorders) were evaluated using a validated MINI structured interview.

Results The overall prevalence of DSP was 50\% in our sample of adolescents. There were no differences between girls and boys in the prevalence of DSP $(p=0.24)$. DSP was significantly higher in those with major depressive disorder $(p=0.018)$, generalized anxiety disorder $(p=0.025)$, and almost significant for obsessive-compulsive disorder $(p=0.055)$. DSP was not more prevalent in hypo-manic disorders, psychotic disorders, or any eating disorders (all p-values $>0.3$ ).

Discussion We found a significant co-morbidity between DSP and several psychiatric disorders. This suggests that there is an added burden of sleep problems in those with issues relating to mental well-being. As our study population is from a 